\title{
A COMPREHENSIVE REVIEW OF A RACE OF VACCINATION AGAINST COVID - 19 VIRUS : THE RECENT VACCINES DEVELOPED
}

\author{
Maimonah Q. Yahya', Zena S. Hamed'2, Seema M. Kasim ${ }^{3}$ \\ ${ }^{1}$ Department of Clinical Laboratory Science, College of Pharmacy, Mosul University, Mosul, Iraq \\ ${ }^{2}$ Department of pharmacology and toxicology, College of Pharmacy, Mosul University, Mosul, Iraq \\ ${ }^{3}$ Department of pharmaceutical chemistry, College of Pharmacy, Mosul University, Mosul, Iraq
}

*Corresponding author: pharm.maymona@uomosul.edu.iq

\begin{abstract}
Vaccines are urgently needed at the present time to protect humanity from COVID-19, which is caused by the Coronavirus 2 that causes severe acute respiratory syndrome. Large quantities of antibodies which have high affinity virus-neutralizing capability were induced by vaccines may optimally prevent infection and avoid unfavourable effects. During the progression of the vaccine, different vaccine models were assessed by disparate groups. This review summarizes the currently approved COVID19 vaccines with late-stage clinical results. The currently authorized SARS-CoV-2 vaccines with late stage clinical data are summarized in this review. Thrombosis may be related in some way to the vaccine, but the direct link has not yet been established. It was stressed that the probability of developing strokes is very small and does not compare with the benefit gained from using the vaccine at all.
\end{abstract}

Keywords: COVID-19, Vaccines, Viral vector vaccine, mRNA vaccine, Inactivated vaccine 


\section{Introduction}

COVID-19 (2019-novel beta coronavirus) is a disease caused by SARS-CoV-2, newly discovered in China and spread to all over the world that reported as epidemics by World Health Organization (WHO)(Zhu et al., 2020). Such disease has emerged in late 2019, which has spread globally with, at time of writing, Overall, 150,110,310 confirmed cases of COVID-19 have been reported to WHO since 2:41 pm CEST on 30 April 2021, including 3,158,792 deaths. A total of 1,011,457,859 doses of vaccines were administered as at 29 April 2021. (World Health Organization,2021). It has caused an Emergency on International Public Health especially among the elderly and those with underlying illnesses who are vulnerable to acute respiratory distress syndrome (ARDS)( Koirala et al., 2020).

Corona viruses are members of the Coronaviridae (subfamily Coronavirinae) family of viruses infecting a wide range of organisms, causing many symptoms depending on host immunity, varying from a flu virus to severe and ultimately fatal illnesses like SARS( severe acute respiratory syndrome ), MERS (middle east respiratory syndrome), and novel coronavirus disease 2019 (2019-nCoV) (Dhama et al., 2020; Boopathi et al., 2020 ). Coronaviruses have single-stranded (ss) positive-sense RNA genome that is not segmented. Nucleocapsid $(N)$, membrane $(M)$, spike $(S)$ and envelop (E) are four structural proteins those with others non-structural proteins (nsp) are the result of encoding the virus genome (Sarma et al., 2020).

Coronavirus S protein attached to ACE2 (angiotensin converting enzyme 2) receptors present on many human cells including lung cells permitting virus entry. Host protease (i.e. furin and trypsin) rupture spike $S$ protein leads to release the fusion peptide which in turn activates membrane fusion mechanism. Blocking virus entry is targeting for treatment option (Bian et al., 2020). The N-protein present inside the capsid has an effective N-terminal that bind with viral RNA causing cells to be hijacked. Once the virus enters the cell, the RNA genetic material of the virus is transferred into the cytoplasm. The replication / transcription complex (RTC), which is made up of non-structural proteins, plays a very important role in viral RTC by coating the viral RNA genome with N-proteins (nsp). Preventing such contact is targeting for effective drug to stop viral replication and transcription (Chowdhury et al., 2020). Finally, special insights are sended from infected cells by transported through smooth vesicles present in the cell membrane and secreted outside the cell via exocytosis process become ready to infect other cells (Dhama et al., 2020).

While certain therapeutic options for COVID-19 have shown efficacy in vitro studies, they have yet to be tested in randomized animal or human clinical trials, limiting their realistic applicability in the current pandemic. (Abubakar et al., 2020). The requirement of SARS-CoV-2 vaccines is urgently needed as a result of its rapid spread and infection between people as well as unavailability of antiviral therapeutic agents. 


\section{Methods}

Since the occurrence of COVID-19 until December 2020, a number of previously published studies from various countries were collected and summarized in this study, with a focus on the discovered vaccines that have already been used in most countries around the world, as well as how these vaccines work in preventing the disease and other details. Studies that included statistics on vaccinated people in each country were excluded.

An evaluation in SARS-CoV-2 patients found that the primary targets for the receptor binding domain of the $S 1$ subunit were binding and neutralizing antimicrobials. The spike protein especially in its original configuration was established, as the virus's immune antigen shortly after SARS-CoV-2 emerged at the end of 2019 (Huang et al., 2020). Once this alleged vaccine target was identified, the next challenge was determining how to obtain the highest possible immune system response to it (the vaccine-induced response that, paradoxically, worsens disease severity in the face of a viral challenge). This response has the following characteristics: neutralizing anticorps, T-cell generative, and immunological disease prevention (the vaccine-induced response that paradoxically increased disease severity upon viral challenge) (Wang et al., 2020).

Various vaccine designs were evaluated by various groups during the development of the SARS-CoV2 vaccine. The following are the currently authorized SARS-CoV-2 vaccines with late-stage clinical data:

\section{1-Viral Vector Vaccines}

\section{A.Sputnik V (Gam-COVID-Vac)}

On August 11, 2020, Russia's President Vladimir Putin was announce the world's first vaccine against the COVID-19 pandemic, which Russia successfully developed after two months testing on human. The vaccine was developed by The Gamaleya National Research Centre for Epidemiology and Microbiology in Moscow. It is known as "Sputnik V," after the first orbital satellite launched in 1957 by the Soviet Union, which sparked the global space race. (Shervani et al., 2020).

The vaccine contains two components, one with the recombinant adenovirus vector based on the human adenovirus type 26 and another with the adenovirus vector based on the human adenovirus type 5, both containing SARS-CoV-2 S protein gene (Rawat et al., 2020). The Russian researchers expect more immune response and more prolonged response by using two different vectors (Uddin et al., 2021). Adenovirus serves as a "shell" for delivering the coronavirus gene to cells and initiating the synthesis of the new envelope made up from coronavirus's proteins, thus exposing the immune system to a possible threat. The Sputnik V (Gam-COVID-Vac) vaccine aims to shorten the time it takes for SARS-CoV-2, the virus that caused the COVID-19 pandemic, to establish immunity(Ahn et al., 2020). The rAd26-S/rAd5-S vaccine regimen consists of an initial Ad26-based SARS-CoV-2 S vaccination accompanied by an Ad5-SARS-CoV-2 S booster vaccination after twenty one days (Lundstrom, 2020). The dose is 1011 viral particles/dose for each recombinant adenovirus (Creech et al., 2021). Storage must be at $\left(-18{ }^{\circ} \mathrm{C}\right.$ in liquid form) but can be stored at $\left(2-8^{\circ} \mathrm{C}\right.$ in freeze dried) up to 6 months (Logunov et al., 2020). 
The results of a phase I and II trial were released a few weeks after the approval, followed by the results of a phase III trial. The efficacy of vaccine was $91.6 \%$ according to interim results from a phase III trial which indicating that In healthy adult volunteers, the vaccine is well tolerated, safe and does not lead to serious health problems (Logunov et al., 2021). It induces strong humoral and cellular immune responses in all healthy volunteers and it is highly immunogenic, with antibody titers in vaccinated participants higher than those in convalescent plasma (Logunov et al.,2020; Klasse et al.,2021). The post-registration phase III trial is currently underway in Russia and Belarus, with over 40000 participants, and the plan is to carry out the phase III trial of Sputnik V in various other countries such as the UAE, India, Venezuela, Egypt, and Brazil (Rawat et al., 2020). At least 20 countries have approved the inoculation for use including Russia, Belarus, Argentina, Serbia, UAE, Algeria, Palestine and Azerbaijan (Logunov et al.,2021;Fairgrieve et al., 2020).

\section{B. AZD1222}

A vaccine based on a chimp adenovirus (ChAdOx1/AZD1222) encoding the spike glycoprotein of SARS-CoV-2 was developed by the British-Swedish company AstraZeneca and the University of Oxford (Uddin et al.,2021; Poland et al., 2020). It is composed of DNA that codes for the S protein and is encased in a capsid from a chimpanzee adenovirus. The adenovirus with the accompanying DNA is brought into the cell by endocytosis, the DNA is released into the cytoplasm, migrates to the cell nucleus where it is transcribed creating mRNA that codes for the $S$ protein. The $S$ protein is processed and presented by the $\mathrm{MHC} I$ and $\mathrm{MHC}$ II complexes by non-immune and immune antigen presenting cells, respectively (Liu, 2019). The dose is $5 \times 1010$ viral particles, given in two doses twenty eight days apart (Creech et al.,2021), storage must be at 2-8 C for 6 months (Logunov et al., 2021). One of the important advantages over Moderna and Pfizer, COVID-19 vaccines of Oxford-AstraZeneca is the fact that it is possible to store and distribute at $2-8^{\circ} \mathrm{C}$ (Nguyen et al., 2020).

A phase I and II trial with 543 individuals receiving the AZD1222 vaccine tested a prime and a primeboost schedule. Phase III were published with an efficacy $82.4 \%$ (Nguyen et al., 2020). The study found that the first dose of vaccine triggered humoral responses, characterized by anti-spike IgG and neutralizing antibodies, as well as IFN T-cell responses in the majority of recipients, and that the second dose of vaccine triggered an additional increase in humoral immune outcomes. (Poland et al., 2020).

Adverse events such as tenderness and pain at the injection site, fever, chills, headache, fatigue, malaise, muscle aches, and nausea were mostly mild and occurred within $4-5$ days of vaccination. The protocol included the use of paracetamol as a preventative measure, which reduced both local and systemic reactions to the vaccine. (Poland et al., 2020). The vaccine has not yet obtained an authorization for emergency use in the USA, but it has been authorized for emergency use in several others. It was added to the list of emergency uses by the World Health Organization in February 2021 (Nguyen et al., 2020). Britain, India and Mexico used such vaccines as an emergency (Longunov et al., 2021; Nguyen et al., 2020). 
The news of the Oxford-AstraZeneca vaccine, which has been linked to blood clots, has made us even more wary (Wise,2021). Thromboembolic events associated with this vaccine were reviewed by the European Medicines Agency's (EMA) Pharmacovigilance Risk Assessment Committee (PRAC). This pandemic has humbled us and continues to present new challenges. A safety signal does not mean that the vaccination campaign should be halted, but it does mean that further investigation is required, as recognized by the EMA. Formal paraphrase According to World Health Organization statements, the specific vaccine has been given a green light; however, these side effects need to be studied further(Wise, 2021).

\section{JNJ- 78436735 or Ad26.COV2.S}

The Janssen Pharmaceutical Companies of Johnson \& Johnson developing a coronavirus vaccine known as JNJ- 78436735 or Ad26.COV2.S at Beth Israel Deaconess Medical Center in Boston (Rai et al., 2021). It is a recombinant, replication-incompetent adenovirus serotype 26 (Ad26) vector vaccine that encodes the stabilized perfusion spike glycoprotein of SARS-CoV-2, the virus responsible for COVID-19(Oliver et al., 2021). Ad26-based vaccines are generally safe and highly immunogenic, and the vaccine was developed from the first clinical isolate of the Wuhan strain(Sadoff et al., 2021). It is given in a single dose $5 \times 1010$ viral particle intramuscularly (Creech et al., 2021). This candidate vaccine requires storage at $\left(-20^{\circ} \mathrm{C}\right.$ ) and can be stored at $2-8^{\circ} \mathrm{C}$ for three months (Logunov et al., 2020; Poland et al., 2020).

Phase III trial showed that the vaccine was $66 \%$ effective overall in the prevention of moderate to intense COVID-19 infection occurs twenty-eight days after vaccination. But it proved less effective in South Africa, where a new variant of the virus has appeared, offering just $57 \%$ protection from moderate to severe infection there ( $95 \%$ of cases were due to infection from the variant). The level of protection in the U.S. was $72 \%$ and it was $66 \%$ in Latin America (Rai et al., 2021; Funk et al., 2021). Ad26.COV2.S vaccine has been showed to be $100 \%$ effective against sever COVID-19 after forty nine days. Emergency use authorization process initiated in USA and Bahrain (Logunov et al., 2021)

Following news that six women out of more than 6.8 million of administered doses in the United States developed rare and severe type of blood clots, 6 to 13 days after receiving the coronavirus vaccine, Johnson \& Johnson has decided to postpone its introduction in Europe (Chadwick, 2021).

\section{2-mRNA Vaccines}

\section{A.BNT162b2:}

A series of mRNA-based COVID-19 vaccines has also been produced by Pfizer and BioNtech (BNT162b1 and BNT162b2). BNT162b2 developed a higher value of T-cell responses in phase I and II trials and had a beneficial safety profile, so it was chosen as the candidate vaccine for evaluation in phase III trials, which are currently enrolling over 44,000 volunteers. (Poland et al., 2020).

All clinical trials in phases I, II, and III were published. BNT162b2 is made up of a nucleoside-modified messenger RNA (modRNA) that encodes an optimized viral full-length spike (S) glycoprotein of the severe acute respiratory syndrome coronavirus 2 (SARS-CoV-2) that serves as the target of virus neutralizing antibodies(Polack et al., 2020). Messenger RNA (mRNA) is encapsulated with lipid 
nanoparticles (LNPs), which allow it to enter host cells by shielding naked mRNA from RNases after injection and enhancing endocytosis into cells surrounding the injection site. Thus, the formulation of LNPs allows for the expression of the $S$ protein as well as the induction of antibody and cellular immune responses(Balakrishnan, 2020). When the lipid nanoparticle is injected, its phospholipid membrane fuses with the host membrane, releasing the mRNA into the cytoplasm of the target cell. The $S$ protein mRNA is then translated at the rough endoplasmic reticulum, resulting in the $S$ protein being present in the cytoplasm. Major Histocompatibility Complex I (MHC I) and II (MHC II) degrade and express the S protein. After binding to the $S$ protein fragment, a T-helper cell releases interleukins, causing $B$ cells to proliferate and differentiate into plasma cells. These plasma cells then produce antibodies against the $S$ protein fragment(Liu, 2019). The dose is $30 \mathrm{~g}$, administered intramuscularly in two doses 21 days apart (Creech et al., 2021). The Pfizer and BioNtech vaccine has unique storage requirements, as it must be kept at $-70^{\circ} \mathrm{C}$ but can also be kept at $2-8^{\circ} \mathrm{C}$ for 30 days or at room temperature for 2 hours (Logunov et al., 2021, Sereviante et al., 2020).

The vaccine was found to be 95 percent effective in preventing COVID-19 in phase III trials involving 43,538 participants, with 170 confirmed cases of COVID-19 in the first twenty-eight days (162 cases observed in the control group versus eight in the vaccinated group). Furthermore, while Pfizer has reported no significant safety concerns to date, the vaccine's medium and long-term safety remains unknown (Badiani et al., 2020). It was authorized by FDA, EMA and UK and current approved in many countries like US, EU and UK (Logunov et al., 2021).

\section{B. mRNA-1273:}

In collaboration with Moderna, the National Institute of Allergy and Infectious Diseases (NIAID) in the United States has begun research to develop mRNA-1273, an mRNA vaccine (Pandey et al., 2020). The nucleoside-modified mRNA vaccine is delivered in the form of lipid particles. It, like the mechanisms of BNT162b2 vaccines, allows delivery of nucleoside-modified mRNA into host cells to enable expression of the SARS-CoV-2 S antigen. Specialized antigen presenting cells (APCs) engulf mRNA virus vaccine and activate $T$ - helper cells which in turn enable $B$ cells to make antibodies that can block the virus from infecting cells and will remember how to fight the virus that causes COVID-19 if infected the body in the future (Meo et al., 2021). Furthermore, it is regarded as reasonably safe because it is composed of neither the inactivated pathogen nor the live pathogen's subunits(Tu et al., 2020). The regimen is $100 \mu \mathrm{g}$ in 2 shots, given twenty eight days apart by injection in the intramuscular-deltoid muscle (Meo et al., 2021). The requirement for a storage temperature of $-20^{\circ} \mathrm{C}$ for 30 days, $2-8^{\circ} \mathrm{C}$ for 30 days, and room temperature for 2 hours is one potential issue for vaccine deployment(Logunov et al., 2021; Poland et al., 2020).

Moderna announced the findings of an interim analysis of their Phase III trial is ongoing involving more than 30,000 participants. All phases I, II and III clinical trials were published. They found their vaccine candidate mRNA-1273 to be safe and well tolerated and to have a vaccine efficacy of $94.5 \%$ (35Pain at the injection site, chills, nausea, myalgia, and fever are both moderate local and systemic side effects that occur within a few days of vaccination (Meo et al., 2021). It was emergency authorized by FDA, 
EMA and UK and current approvals in US, EU, Canada, Israel, Switzerland and UK (Logunov et al., 2021).

\section{3- Inactivated Coronavirus vaccine: \\ CoronaVac and BBIBP Cor-V}

China National Biotec Group (CNBG), a joint venture between Sinopharm, a state-owned corporation, and Sinovac, a private Chinese company, is well ahead of the curve in coronavirus vaccine trials (Uddin, 2021).

Sinopharm has created two whole-virus inactivated vaccines. The Wuhan Institute of Biological Products created the first vaccine candidate, New Crown COVID-19 (inactivated CNO2 strain of SARSCoV-2 created from Vero cells). The Beijing Institute of Biological Products developed the second vaccine candidate, BBIBP Cor-V (inactivated HBO2 strain of SARS-CoV-2 created from Vero cells)(Logunov et al., 2020; Poland et al, 2020). Sinovac Biotech developed an inactivated vaccine called (CoronaVac.) (Uddin, 2021).

Inactivated vaccines are viruses that have been physiochemically treated to reduce their pathogenicity. When inactivated viruses are injected, they are engulfed by antigen-presenting cells, and different epitopes are presented to the immune system. They also have a good safety profile because the virus cannot cause disease; however, they require a booster strategy to develop immune memory(Yu et $\mathrm{al}, 2020)$. The vaccines are given in two doses intramuscularly with aluminum hydroxide adjuvant. New Crown COVID-19 vaccine dose is $5 \mu \mathrm{g}$ in 2 doses. BBIBP Cor-V vaccine dose is $4 \mu \mathrm{g}$ in 2 doses twenty one days apart, while Corona-vac vaccine dose is $3 \mu \mathrm{g}$ in 2 doses fourteen days apart (Creech et al., 2021). One potential issue with vaccine deployment is that it requires a storage temperature of 2-8 C; the lifespan is unknown(Creech et al., 2021).

The New Crown COVID-19 vaccine has been shown to be safe and immunogenic in a randomized, double-blind and placebo controlled phase I and II trials that published with no phase III published data (40). Its efficacy is $86 \%$ in UAE with 31000 volunteers and $70 \%$ in China. It was approved in China, UAE, Bahrain, Serbia, Peru, Zimbabwe and Morocco with limited use in China, Egypt, Jordan and UAE (Logunov et al., 2021; Wise, 2021). While the efficacy of Corona-vac is $50.38 \%$ in Brazil, $91 \%$ in Turkey and $65 \%$ in Indonesia(Poland et al., 2020). It is currently approved for use in Brazil, Uruguay, Columbia, Bolivia, China, Turkey, Azerbaijan and Indonesia with limited use in China and Indonesia and emergency use in Turkey(Logunov et al., 2021;Poland et al., 2020). The adverse effects of these vaccines may include localize pain, headache, muscle ache, and hyperthermia (Logunov et al., 2021).

\section{Conclusion}

Vaccines are an important component of public health and have been shown to prevent serious diseases. They, like any other medications, have the ability to cause harmful effects which must be balanced against the likelihood of developing a serious or even fatal disease like COVID-19. Your participation by taking the vaccine will increase the safety of COVID-19 vaccines evaluation. But, using a face mask, good hand hygiene, and maintaining a safe physical distance are still important. The greater the similarity between the vaccine action and the immune system triggering action, the more effective and multi-step protective mechanisms the immune system is developing and provides multilevel protection, increase the effectiveness of the vaccine. The European Medicines Agency (EMA) 
indicated that clots can be considered a very rare side effect of the vaccine, and confirmed that the benefits of using the vaccine are much greater than the risks of the Corona virus, and that it will continue to recommend its use according to controls.

\section{Recommendations for further studies}

Suggestions for further investigation Despite the fact that many well-designed ongoing clinical trials are nearing completion, randomized clinical trials on the effectiveness of vaccine use in COVID-19 are desperately needed.

\section{Conflicts of Interest}

The author declares no conflicts of interest.

\section{References}

- Abubakar, A. R., Sani, I. H., Godman, B., Kumar, S., Islam, S., Jahan, I., \& Haque, M. (2020). Systematic Review on the Therapeutic Options for COVID-19: Clinical Evidence of Drug Efficacy and Implications. Infection and drug resistance, 13, 4673. https://doi.org/10.2147/IDR.S289037

- Ahn, D. G., Shin, H. J., Kim, M. H., Lee, S., Kim, H. S., Myoung, J., ... \& Kim, S. J. (2020). Current status of epidemiology, diagnosis, therapeutics, and vaccines for novel coronavirus disease 2019 (COVID-19). Journal of microbiology and biotechnology, 30(3), 313-324. https://doi.org/10.4014/jmb.2003.03011.

- Badiani, A. A., Patel, J. A., Ziolkowski, K., \& Nielsen, F. B. H. (2020). Pfizer: The miracle vaccine for COVID-19?. Public Health in Practice, 1, 100061.https://doi.org/10.1016/j.puhip.2020.100061

- Balakrishnan, V. S. (2020). The arrival of Sputnik V. The Lancet Infectious Diseases, 20(10), 1128. https://doi:10.3390/vaccines8030404.

- Bian, J., \& Li, Z. (2020). Angiotensin-converting enzyme 2 (ACE2): SARS-CoV-2 receptor and RAS modulator. Acta Pharmaceutica Sinica B. https://doi.org/10.1016/j.apsb.2020.10.006.

- Boopathi, S., Poma, A. B., \& Kolandaivel, P. (2020). Novel 2019 coronavirus structure, mechanism of action, antiviral drug promises and rule out against its treatment. Journal of Biomolecular Structure and Dynamics, 1-10. https://doi.org/10.1080/07391102.2020.1758788.

- Chadwick L. (2021). Johnson \& Johnson says it is delaying rollout of its COVID vaccine in Europe amid blood clot reports. Use. https://www.euronews.com [online]. https://www.euronews.com/amp/2021/04/13/us-recommends-johnson-johnson-covid-vaccinepause-due-to-bloodclots?fbclid=IwAR33PIRaFb0pt7fzCdAdbIAnqeNGPm9wk311dqgN20aUJuphrK-dbwjL4Y\#referrer=https://www.google.com\&csi=0

- Chowdhury, M. A., Hossain, N., Kashem, M. A., Shahid, M. A., \& Alam, A. (2020). Immune response in COVID-19: A review. Journal of Infection and Public Health. doi: 10.1016/j.apsb.2020.10.006.

- Creech, C. B., Walker, S. C., \& Samuels, R. J. (2021). SARS-CoV-2 vaccines. Jama, 325(13), 1318-1320.https://doi:10.1001/jama.2021.3199.

- $\quad$ Dhama, K., Khan, S., Tiwari, R., Sircar, S., Bhat, S., Malik, Y. S., ... \& Rodriguez-Morales, A. J. (2020). Coronavirus disease 2019-COVID-19. Clinical microbiology reviews, 33(4). DOI: 10.1128/CMR.00028-20.

- Fairgrieve, D., Feldschreiber, P., Howells, G., \& Pilgerstorfer, M. (2020). Products in a Pandemic: Liability for Medical Products and the Fight against COVID-19. European Journal of Risk Regulation, 11(3), 565-603. DOI: https://doi.org/10.1017/err.2020.54

- Funk, C. D., Laferrière, C., \& Ardakani, A. (2021). Target Product Profile Analysis of COVID-19 Vaccines in Phase III Clinical Trials and Beyond: An Early 2021 Perspective. Viruses, 13(3), 418. https://doi.org/10.3390/v13030418

- World Health organization.(2021). WHO Coronavirus (COVID-19) Dashboard. Available online 
at: https://covid19.who.int/ ( accessed April 30, 2021).

- Huang, Y., Yang, C., Xu, X. F., Xu, W., \& Liu, S. W. (2020). Structural and functional properties of SARS-CoV-2 spike protein: potential antivirus drug development for COVID-19. Acta Pharmacologica Sinica, 41(9), 1141-1149.

- Klasse, P. J., Nixon, D. F., \& Moore, J. P. (2021). Immunogenicity of clinically relevant SARSCoV-2 vaccines in nonhuman primates and humans. Science Advances, 7(12), eabe8065. DOI: 10.1126/sciadv.abe8065

- Koirala, A., Joo, Y. J., Khatami, A., Chiu, C., \& Britton, P. N. (2020). Vaccines for COVID-19: The current state of play. Paediatric respiratory reviews, 35, 43-49. https://doi.org/10.1016/j.prrv.2020.06.010.

- Liu, M. A. (2019). A comparison of plasmid DNA and mRNA as vaccine technologies. Vaccines, 7(2), 37. https://doi:10.3390/vaccines7020037

- Logunov, D. Y., Dolzhikova, I. V., Shcheblyakov, D. V., Tukhvatulin, A. I., Zubkova, O. V., Dzharullaeva, A. S., ... \& Gam-COVID-Vac Vaccine Trial Group. (2021). Safety and efficacy of an rAd26 and rAd5 vector-based heterologous prime-boost COVID-19 vaccine: an interim analysis of a randomised controlled phase 3 trial in Russia. The Lancet, 397(10275), 671-681. https://doi.org/10.1016/S0140-6736(21)00234-8

- Logunov, D. Y., Dolzhikova, I. V., Zubkova, O. V., Tukhvatullin, A. I., Shcheblyakov, D. V., Dzharullaeva, A. S., ... \& Gintsburg, A. L. (2020). Safety and immunogenicity of an rAd26 and rAd5 vector-based heterologous prime-boost COVID-19 vaccine in two formulations: two open, non-randomised phase 1/2 studies from Russia. The Lancet, 396(10255), 887-897. https://doi.org/10.1016/S0140-6736(20)31866-3

- Lundstrom, K. (2020). Application of Viral Vectors for Vaccine Development with a Special Emphasis on COVID-19. Viruses, 12(11), 1324. https://doi:10.3390/v12111324.

- Meo, S. A., Bukhari, I. A., Akram, J., Meo, A. S., \& Klonoff, D. C. (2021). COVID-19 vaccines: comparison of biological, pharmacological characteristics and adverse effects of Pfizer/BioNTech and Moderna Vaccines. Eur Rev Med Pharmacol Sci,25(3),1663-69. https//doi: 10.26355/eurrev_202102_24877.

- Nguyen, L. C., Bakerlee, C. W., McKelvey, T. G., Rose, S. M., Norman, A. J., Joseph, N., ... \& Morrison, J. (2020). Evaluating use cases for human challenge trials in accelerating SARSCoV-2 vaccine development. Clinical Infectious Diseases. https://doi.org/10.1093/cid/ciaa935.

- Oliver, S. E., Gargano, J. W., Scobie, H., Wallace, M., Hadler, S. C., Leung, J., ... \& Dooling, K. (2021). The Advisory Committee on Immunization Practices' Interim Recommendation for Use of Janssen COVID-19 Vaccine-United States, February 2021. Morbidity and Mortality Weekly Report, 70(9), 329. doi: 10.15585/mmwr.mm7009e4.

- Pandey, S. C., Pande, V., Sati, D., Upreti, S., \& Samant, M. (2020). Vaccination strategies to combat novel corona virus SARS-CoV-2. Life sciences, 117956. https://doi.org/10.1016/j.lfs.2020.117956.

- Polack, F. P., Thomas, S. J., Kitchin, N., Absalon, J., Gurtman, A., Lockhart, S., ... \& Gruber, W. C. (2020). Safety and efficacy of the BNT162b2 mRNA Covid-19 vaccine. New England Journal of Medicine, 383(27), 2603-2615.DOI: 10.1056/NEJMoa2034577

- Poland, G. A., Ovsyannikova, I. G., \& Kennedy, R. B. (2020). SARS-CoV-2 immunity: review and applications to phase 3 vaccine candidates. The Lancet. https://doi.org/10.1016/S01406736(20)32137-1

- Rai, N. K., Ashok, A., \& Akondi, B. R. (2021). Journey from Coronavirus Pandemic to Vaccines. Asian Journal of Pharmaceutical Research and Health Care, 13(1), 1-3. DOI: 10.18311/ajprhc/2021/27078.

- Rawat, K., Kumari, P., \& Saha, L. (2020). COVID-19 vaccine: A recent update in pipeline vaccines, their design and development strategies. European journal of pharmacology, 173751. https://doi.org/10.1016/j.ejphar.2020.173751.

- Rodriguez Coira, J., \& Sokolowska, M. (2020). SARS-CoV-2 candidate vaccines-composition, mechanisms of action and stages of clinical development. Allergy. https://doi.org/10.1111/all.14714.

- Sadoff, J., Le Gars, M., Shukarev, G., Heerwegh, D., Truyers, C., de Groot, A. M., ... \& Schuitemaker, H. (2021). Interim Results of a Phase 1-2a Trial of Ad26. COV2. S Covid-19 Vaccine. New England Journal of Medicine. http://doi:10.1056/NEJMoa2034201

- Sarma, P., Shekhar, N., Prajapat, M., Avti, P., Kaur, H., Kumar, S., ... \& Medhi, B. (2020). Insilico homology assisted identification of inhibitor of RNA binding against 2019-nCoV N-protein ( $\mathrm{N}$ terminal domain). Journal of Biomolecular Structure and Dynamics, 1-9. 
https://doi.org/10.1080/07391102.2020.1753580.

- Seneviratne, S. L., Jayarajah, U., Abeysuriya, V., Rahman, A., \& Wanigasuriya, K. (2020). COVID-19 vaccine landscape. Journal of the Ceylon College of Physicians, 51(2). DOI: http://doi.org/10.4038/jccp.v51i2.7906

- Shervani, Z., Khan, I., Khan, T., \& Qazi, U. Y. (2020). COVID-19 Vaccine. Advances in Infectious Diseases, 10(03), 195. https://doi.org/10.4236/aid.2020.103020.

- Tu, Y. F., Chien, C. S., Yarmishyn, A. A., Lin, Y. Y., Luo, Y. H., Lin, Y. T., ... \& Chiou, S. H. (2020). A review of SARS-CoV-2 and the ongoing clinical trials. International journal of molecular sciences, 21(7), 2657. https:doi:10.3390/ijms21072657.

- Uddin, K. N. (2021). Corona vaccine. BIRDEM Medical Journal, 11(1), 1-5. https://doi.10.3329/birdem.v11i1.51038.

- Wang, M. Y., Zhao, R., Gao, L. J., Gao, X. F., Wang, D. P., \& Cao, J. M. (2020). SARS-CoV-2: structure, biology, and structure-based therapeutics development. Frontiers in cellular and infection microbiology, 10. doi: 10.3389/fcimb.2020.587269.

- Wise, J. (2021). Covid-19: European countries suspend use of Oxford-AstraZeneca vaccine after reports of blood clots. doi: https://doi.org/10.1136/bmj.n699

- Zhu, N., Zhang, D., Wang, W., Li, X., Yang, B., Song, J., ... \& Tan, W. (2020). A novel coronavirus from patients with pneumonia in China, 2019. New England journal of medicine. DOI: 10.1056/NEJMoa2001017. 